TRANSACTIONS OF THE

AMERICAN MATHEMATICAL SOCIETY

Volume 363, Number 12, December 2011, Pages 6481-6503

S 0002-9947(2011)05294-3

Article electronically published on July 26, 2011

\title{
GENERALIZED FRACTIONAL INTEGRAL OPERATORS AND FRACTIONAL MAXIMAL OPERATORS IN THE FRAMEWORK OF MORREY SPACES
}

\author{
YOSHIHIRO SAWANO, SATOKO SUGANO, AND HITOSHI TANAKA
}

\begin{abstract}
The action of the generalized fractional integral operators and the generalized fractional maximal operators is investigated in the framework of Morrey spaces. A typical property of the functions which belongs to Morrey spaces under pointwise multiplication by the generalized fractional integral operators and the generalized fractional maximal operators is established. The boundedness property of the fractional integral operators on the predual of Morrey spaces is shown as well. A counterexample concerning the FeffermanPhong inequality is given by the use of the characteristic function of the Cantor set.
\end{abstract}

\section{INTRODUCTION}

The purpose of the present paper is to study certain estimates related to the generalized fractional integral operator, defined by

$$
T_{\rho} f(x)=\int_{\mathbb{R}^{n}} f(y) \frac{\rho(|x-y|)}{|x-y|^{n}} d y,
$$

where $\rho:[0, \infty) \rightarrow[0, \infty)$ is a suitable function, and to the generalized fractional maximal operator, defined by

$$
M_{\rho} f(x)=\sup _{x \in Q \in \mathcal{Q}} \frac{\rho(\ell(Q))}{|Q|} \int_{Q}|f(y)| d y,
$$

in the framework of Morrey spaces. Here, we use the notation $\mathcal{Q}$ to denote the family of all cubes in $\mathbb{R}^{n}$ with sides parallel to the coordinate axes, $\ell(Q)$ to denote the sidelength of $Q$ and $|Q|$ to denote the volume of $Q$. If $\rho(t) \equiv t^{n \alpha}, 0<\alpha<$ 1 , the operators $T_{\rho}$ and $M_{\rho}$ are the usual fractional integral operator (the Riesz potential) and the usual fractional maximal operator, which we write as $I_{\alpha}$ and $M_{\alpha}$, respectively. The fractional integral operator is one of the important tools in harmonic analysis with a background in partial differential equations. In fact, for a nice function $f$ on $\mathbb{R}^{n}$, the solution of Laplace equation $\Delta u=f$ can be represented by using a fractional integral operator (cf. [19]).

Received by the editors July 16, 2008 and, in revised form, December 22, 2009.

2010 Mathematics Subject Classification. Primary 42B35; Secondary 42B25.

Key words and phrases. Generalized fractional integral operator, generalized fractional maximal operator, Morrey space, Olsen's inequality, block space.

The third author was supported by the Global COE program at the Graduate School of Mathematical Sciences at the University of Tokyo, and was also supported by the Fūjyukai Foundation. 
The integral kernel of $I_{\alpha},|y|^{n \alpha} /|y|^{n}$, has singularity at zero and infinity. One could expect more singularity to the integral kernel $T_{\rho}, \rho(|y|) /|y|^{n}$, for example, up to logarithmic factors. However, it is impossible to add any more singularity in the framework of the (ordinal) Lebesgue spaces. By use of the other function spaces that cover the Lebesgue spaces, the theory of the generalized fractional integral operator $T_{\rho}$ has been developed by many authors who have focused their interest mainly on giving the sufficient conditions in order to obtain the boundedness of $T_{\rho}$ on those spaces (see 6, 7, 8, 12, 13, 14, ).

Morrey spaces, which were introduced by C. Morrey in order to study regularity questions which appear in the Calculus of Variations, describe local regularity more precisely than Lebesgue spaces and widely use not only harmonic analysis but also partial differential equations (cf. 10]). To define Morrey spaces we recall some definitions and notation.

All cubes are assumed to have their sides parallel to the coordinate axes. We denote by $\mathcal{Q}$ the family of all cubes in $\mathbb{R}^{n}$. For $Q \in \mathcal{Q}$ we use the notation $\ell(Q)$ to denote the sidelength of $Q$ and $c Q$ to denote a cube with the same center as $Q$, but with sidelength $c \ell(Q)$. $|E|$ denotes the Lebesgue measure of $E \subset \mathbb{R}^{n}$.

Let $0<p<\infty$ and $\phi:[0, \infty) \rightarrow[0, \infty)$ be a suitable function. For a function $f$ locally in $L^{p}\left(\mathbb{R}^{n}\right)$ we set

$$
\|f\|_{p, \phi}=\sup _{Q \in \mathcal{Q}} \phi(\ell(Q))\left(\frac{1}{|Q|} \int_{Q}|f(x)|^{p} d x\right)^{1 / p} .
$$

We will call the Morrey space $\mathcal{M}^{p, \phi}\left(\mathbb{R}^{n}\right)=\mathcal{M}^{p, \phi}$ the subset of all functions $f$ locally in $L^{p}\left(\mathbb{R}^{n}\right)$ for which $\|f\|_{\mathcal{M}^{p, \phi}}=\|f\|_{p, \phi}$ is finite. Applying Hölder's inequality to (1.1), we see that $\|f\|_{p_{1}, \phi} \geq\|f\|_{p_{2}, \phi}$, provided that $p_{1} \geq p_{2}>0$. This tells us that $\mathcal{M}^{p_{1}, \phi} \subset \mathcal{M}^{p_{2}, \phi}$ when $p_{1} \geq p_{2}>0$.

We now claim that without loss of generality we may assume

$$
\phi(t) \text { is nondecreasing and } \phi(t)^{p} t^{-n} \text { is nonincreasing. }
$$

Indeed, if we let

$$
\phi_{1}(t)=\sup _{t^{\prime} \in[0, t]} \phi\left(t^{\prime}\right)
$$

then

$$
\|f\|_{p, \phi} \leq\|f\|_{p, \phi_{1}} \leq 2^{n / p}\|f\|_{p, \phi} .
$$

This holds by using the simple geometric fact that for any cubes $Q \in \mathcal{Q}$ and any positive numbers $t^{\prime} \leq \ell(Q)$,

$$
\frac{1}{|Q|} \int_{Q}|f(x)|^{p} d x \leq 2^{n} \sup _{Q^{\prime} \in \mathcal{Q}: Q^{\prime} \subset Q, \ell\left(Q^{\prime}\right)=t^{\prime}} \frac{1}{\left|Q^{\prime}\right|} \int_{Q^{\prime}}|f(x)|^{p} d x .
$$

Next, if we let

$$
\phi_{2}(t)=t^{n / p} \sup _{t^{\prime} \geq t} \phi\left(t^{\prime}\right) t^{-n / p}
$$

then $\|f\|_{p, \phi}=\|f\|_{p, \phi_{2}}$. Hereafter, we always assume that $\phi$ satisfies (1.2).

If $\phi(t) \equiv t^{n / p_{0}}, p_{0} \geq p, \mathcal{M}^{p, \phi}$ is the usual Morrey space, and we write this for $\mathcal{M}^{p, p_{0}}$ and the norm for $\|\cdot\|_{\mathcal{M}^{p, p_{0}}}$. Then we have the inclusion

$$
L^{p_{0}}=\mathcal{M}^{p_{0}, p_{0}} \subset \mathcal{M}^{p_{1}, p_{0}} \subset \mathcal{M}^{p_{2}, p_{0}}
$$

when $p_{0} \geq p_{1} \geq p_{2}>0$. 
A classical result for the fractional integral operator $I_{\alpha}$, which is known as the Hardy-Littlewood-Sobolev inequality, is that it is bounded from $L^{p}$ to $L^{q}$ provided that $1 / q=1 / p-\alpha$ (cf. [19]). More generally, $I_{\alpha}$ is bounded from the Morrey space $\mathcal{M}^{p, p_{0}}$ to $\mathcal{M}^{q, q_{0}}$ provided that $1<p \leq p_{0}<\infty, 1<q \leq q_{0}<\infty, 1 / q_{0}=1 / p_{0}-\alpha$ and $q / q_{0}=p / p_{0}$. This result was first due to Adams [1, Theorem 1.3] and was reproved by Chiarenza and Frasca [3, Theorem 2]. If $q / q_{0}=p / p_{0}$ is replaced by $1 / q=1 / p-\alpha$, then, since $f$ in $\mathcal{M}^{p, p_{0}}$ implies $f$ locally in $L^{p}$, using the HardyLittlewood-Sobolev inequality locally and taking care of the larger scales by cruder estimates, one has a naive bound for $I_{\alpha}$ (cf. [15, Theorem 1]).

For a nice function $f$ in $\mathbb{R}^{n}, n>2$, and a weight $w \in \mathcal{M}^{p, n / 2}, 1<p \leq n / 2$, it holds that

$$
\int_{\mathbb{R}^{n}}|f(x)|^{2} w(x) d x \leq C\|w\|_{\mathcal{M}^{p, n / 2}} \int_{\mathbb{R}^{n}}|\nabla f|(x)^{2} d x .
$$

This is the so-called Fefferman-Phong inequality obtained in [9] and has been widely used in partial differential equations. In [15, Olsen obtained an interesting inequality concerning fractional integral operators on the Morrey spaces, which can be understood as a sort of Fefferman-Phong inequality since one has (cf. [19, p. 125])

$$
|f(x)| \leq C \sum_{j=1}^{n} \int_{\mathbb{R}^{n}}\left|\frac{\partial f}{\partial x_{j}}(x-y)\right||y|^{-n+1} d y .
$$

The original proof due to Olsen is somehow complicated and implicitly involves the Calderón-Zygmund decomposition. In [20, the second and third authors gave an alternative proof based on a variant of the good- $\lambda$ inequality of Fefferman and Stein introduced in [5]. In [21], the third author gave another simple proof and extended the result to vector-valued functions. His proof is based on the idea of C. Pérez introduceed in [17] (cf. Lemma 2.1). In the present paper, we introduce Olsen's inequality for the generalized fractional integral operator $T_{\rho}$ (Theorem 1.1) and for the generalized fractional maximal operator $M_{\rho}$ (Theorem 1.7). As a corollary (Corollary 1.2), we have a boundedness property of $T_{\rho}$ on the Morrey spaces, which is closely related to the theorem due to Eridani, Gunawan and Nakai 8 , Theorem $3.1]$.

It is evident that $M_{\alpha} f(x) \leq C I_{\alpha} f(x)$ for all nonnegative functions $f$ due to the estimate

$$
\frac{r^{n \alpha}}{r^{n}} \int_{|y-x|<r} f(y) d y \leq I_{\alpha} f(x), \quad x \in \mathbb{R}^{n}, r>0 .
$$

However, as the examples $f(y)=|y|^{-n \alpha}$ and $x=0$ show, the reverse inequality is false. In view of this, it is a significant relation between $I_{\alpha}$ and $M_{\alpha}$ that

$$
\int_{\mathbb{R}^{n}}\left|I_{\alpha} f(x)\right|^{p} d x \leq C \int_{\mathbb{R}^{n}} M_{\alpha} f(x)^{p} d x, \quad p>1 .
$$

This relation can be proved by use of the good- $\lambda$ inequality of Fefferman and Stein. In [2, motivated by the development of the theory of capacities for potentials of functions of the Morrey space, Adams and Xiao extended this relation to the Morrey space $\mathcal{M}^{p, p_{0}}$. In [21, the third author extended it further to the vector-valued functions. In the present paper, concerning (1.4), we introduce some relations between the generalized fractional operator and the generalized fractional maximal 
operator in the framework of the Morrey spaces $\mathcal{M}^{p, \phi}$ (Theorem 1.3 ). Theorem 1.3 is a generalization of the result in [21, which was found when analysing the proof of Olsens's inequality. Theorem 1.3 and a boundedness property of the generalized fractional maximal operator (Lemma 2.6) give us a generalization of Olsen's inequality (Theorem 1.1).

In the third section, we prove a dual version of Olsen's inequality on the predual of Morrey spaces (Theorem 3.1). As a corollary (Corollary 3.2), we have the boundedness properties of the operator $T_{\rho}$ on the predual of a Morrey space. The results are new even for $I_{\alpha}$ as far as we know. In the last section, we discuss the optimality of our estimates for the fractional integral operator (the Riesz potential) $I_{\alpha}$ and give an interesting counterexample concerning to the Fefferman-Phong inequality by use of the characteristic function of the Cantor set.

The Hardy-Littlewood maximal operator $M$ is defined by

$$
M f(x)=\sup _{x \in Q \in \mathcal{Q}} \frac{1}{|Q|} \int_{Q}|f(y)| d y .
$$

It is well known that the Hardy-Littlewood maximal operator $M$ is bounded on the Morrey space $\mathcal{M}^{p, p_{0}}$ with $1<p \leq p_{0}<\infty$ (see [3, Theorem 1]). A boundedness property of the Hardy-Littlewood maximal operator $M$ on the Morrey space $\mathcal{M}^{p, \phi}$, $p>1$, was first proved by Nakai [1]. Our new result in Lemma 2.4 will cover his result, and we shall not postulate a superfluous assumption in [11].

Following [8], in the definition of $T_{\rho}$ we always postulate the Dini and the doubling conditions on $\rho$.

(1) $\rho$ satisfies the Dini condition

$$
\int_{0}^{1} \frac{\rho(s)}{s} d s<\infty
$$

(2) $\rho$ satisfies the doubling condition

$$
\frac{1}{C_{1}} \leq \frac{\rho(s)}{\rho(t)} \leq C_{1} \text { if } \frac{1}{2} \leq \frac{s}{t} \leq 2 .
$$

A function $\rho$ satisfying (1.6) is said to satisfy the doubling condition (with a doubling constant $C_{1}$ ). We notice that, satisfying (1.2), $\phi$ satisfies the doubling condition (with a doubling constant $2^{n / p}$ ). If $\rho$ satisfies the doubling condition, then

$$
\frac{\log 2}{C_{1}} \rho(t) \leq \int_{t / 2}^{t} \frac{\rho(s)}{s} d s \leq \log 2 \cdot C_{1} \rho(t) \text { for all } t>0 .
$$

We define for $\rho$ satisfying (1.5) and (1.6)

$$
\tilde{\rho}(t)=\int_{0}^{t} \frac{\rho(s)}{s} d s .
$$

We now state our main theorems.

Theorem 1.1. Let $1<p<\infty, q>r, 0 \leq b \leq 1, a>1$ and $(a+b-1) r=a p$. Suppose that $\rho$ satisfies (1.5), (1.6) and that $\tilde{\rho}(t)^{\max (a p, b q)} t^{-n}$ is nonincreasing 1

\footnotetext{
${ }^{1}$ The fact that condition $\tilde{\rho}(t)^{\max (a p, b q)} t^{-n}$ is nonincreasing implies that $\tilde{\rho}(t)^{a p} t^{-n}$ and $\tilde{\rho}(t)^{b q} t^{-n}$ are nonincreasing, since

$$
\tilde{\rho}(t)^{\min (a p, b q)} t^{-n}=\tilde{\rho}(t)^{\min (a p, b q)-\max (a p, b q)} \cdot \tilde{\rho}(t)^{\max (a p, b q)} t^{-n} .
$$
}


Then

$$
\left\|g \cdot T_{\rho} f\right\|_{r, \tilde{\rho}^{a+b-1}} \leq C\|g\|_{q, \tilde{\rho}^{b}}\|f\|_{p, \tilde{\rho}^{a}}
$$

where the constant $C$ is independent of $f$ and $g$.

Theorem 1.1]is a generalization of [15, Theorem 2] and [20, Theorem 1]. Theorem 1.1 is no longer true when $q=r$ (see Proposition 4.1).

Letting $b=0$ and $g \equiv 1$ in Theorem [1.1, we have the following:

Corollary 1.2. Let $1<p<\infty, a>1$ and $(a-1) r=a p$. Then

$$
\left\|T_{\rho} f\right\|_{r, \tilde{\rho}^{a-1}} \leq C\|f\|_{p, \tilde{\rho}^{a}} .
$$

Corollary 1.2 is a generalization of [1, Theorem 1.3].

Theorem 1.3. Let $1 \leq p<\infty,\left\{\begin{array}{l}p \leq q \text { if } p=1, \quad 0 \leq b \leq 1 \text { and } b<a \text {. Suppose } \\ p<q \text { if } p>1,\end{array}\right.$ that $\rho$ satisfies (1.5), (1.6) and that $\tilde{\rho}(t)^{\max (a p, b q)} t^{-n}$ is nonincreasing. Then

$$
\left\|g \cdot T_{\rho} f\right\|_{p, \tilde{\rho}^{a}} \leq C\|g\|_{q, \tilde{\rho}^{b}}\left\|M_{\tilde{\rho}^{1-b}} f\right\|_{p, \tilde{\rho}^{a}},
$$

where the constant $C$ is independent of $f$ and $g$.

Corollary 1.4. Let $1 \leq p<\infty$ and $a>0$. Then

$$
\left\|T_{\rho} f\right\|_{p, \tilde{\rho}^{a}} \leq C\left\|M_{\tilde{\rho}} f\right\|_{p, \tilde{\rho}^{a}} .
$$

Corollary 1.4 is a generalization of [2, Theorem 4.2].

Letting $b=1$ in Theorem 1.3 we have the following corollary as well.

Corollary 1.5. Let $1 \leq p<\infty,\left\{\begin{array}{l}p \leq q \text { if } p=1, \\ p<q \text { if } p>1,\end{array}\right.$ and $a>1$. Then

$$
\left\|g \cdot T_{\rho} f\right\|_{p, \tilde{\rho}^{a}} \leq C\|g\|_{q, \tilde{\rho}}\|M f\|_{p, \tilde{\rho}^{a}} .
$$

Remark 1.6. The functions belonging to the Morrey space have been used as weights in the Fefferman-Phong inequality. Corollary 1.5 would be interesting to give a better understanding of this fact, since the right-hand side contains no $\rho$ factors.

It would be interesting to compare Theorem 1.3 with the following Theorem 1.7

Theorem 1.7. Let $0<p<\infty, 0 \leq b \leq 1$ and $b \leq a$. Suppose that $\rho$ is nondecreasing and $\rho(t)^{a p} t^{-n}$ is nonincreasing. Then

$$
\left\|g \cdot M_{\rho} f\right\|_{p, \rho^{a}} \leq C\|g\|_{p, \rho^{b}}\left\|M_{\rho^{1-b}} f\right\|_{p, \rho^{a}},
$$

where the constant $C$ is independent of $f$ and $g$.

We restate Theorem 1.1 in terms of the fractional integral operator $I_{\alpha}$. The result holds by letting $\rho(t) \equiv t^{n \alpha}, a \alpha=1 / p_{0}$ and $b \alpha=1 / q_{0}$.

Proposition 1.8. Let $0<\alpha<1,1<p \leq p_{0}<\infty, 1<q \leq q_{0} \leq \infty$ and $1<r \leq r_{0}<\infty$. Suppose that $q>r, 1 / p_{0}>\alpha, 1 / q_{0} \leq \alpha, 1 / r_{0}=1 / q_{0}+1 / p_{0}-\alpha$ and $r / r_{0}=p / p_{0}$. Then

$$
\left\|g \cdot I_{\alpha} f\right\|_{\mathcal{M}^{r, r_{0}}} \leq C\|g\|_{\mathcal{M}^{q, q_{0}}}\|f\|_{\mathcal{M}^{p, p_{0}}}
$$

where the constant $C$ is independent of $f$ and $g$. 
Remark 1.9. Our result is stronger than that of Olsen. In fact, Olsen's theorem [15, Theorem 2] is stated with $r / r_{0}=p / p_{0}$ replaced by $1 / r=1 / q_{0}+1 / p-\alpha$. Then the target spaces of our result are strictly smaller than that of Olsen by noticing the inclusion (1.3). A simple calculation suffices to verify this fact: If $r / r_{0}=p / p_{0}$ and $1 / r_{0}=1 / q_{0}+1 / p_{0}-\alpha$, then

$$
\frac{1}{r}=\frac{1}{r_{0}} \frac{p_{0}}{p}=\left(\frac{1}{q_{0}}+\frac{1}{p_{0}}-\alpha\right) \frac{p_{0}}{p}=\left(\frac{1}{q_{0}}-\alpha\right) \frac{p_{0}}{p}+\frac{1}{p} \leq \frac{1}{q_{0}}+\frac{1}{p}-\alpha,
$$

where we have used the fact that $1 / q_{0}-\alpha \leq 0$ and $p_{0} / p \geq 1$.

Remark 1.10. If $r / r_{0}=p / p_{0}=q / q_{0}$, then our result can be proved by using Hölder's inequality. Indeed, the boundedness property of $I_{\alpha}$ on the Morrey space gives us that

$$
\left\|I_{\alpha} f\right\|_{\mathcal{M}^{s, s_{0}}} \leq C\|f\|_{\mathcal{M}^{p, p_{0}}},
$$

where $1 / s_{0}=1 / p_{0}-\alpha$ and $1 / s=p_{0} /\left(p s_{0}\right)$. If $r / r_{0}=p / p_{0}=q / q_{0}$ and $1 / r_{0}=$ $1 / q_{0}+1 / p_{0}-\alpha$, we also have

$$
1 / r_{0}=1 / q_{0}+1 / s_{0} \text { and } 1 / r=1 / q+1 / p .
$$

\section{Proof of the theorems}

The letter $C$ will be used for constants that may change from one occurrence to another. Constants with subscripts, such as $C_{1}, C_{2}$, do not change in different occurrences. For any $1<p<\infty$ we will write $p^{\prime}$ for the conjugate number defined by $1 / p+1 / p^{\prime}=1$. Hereafter, for the sake of simplicity, for any $Q \in \mathcal{Q}$ and $0<p<\infty$ we will write

$$
m_{Q}(f)=\frac{1}{|Q|} \int_{Q} f(x) d x \text { and } m_{Q}^{(p)}(f)=m_{Q}\left(|f|^{p}\right)^{1 / p} .
$$

2.1. Proof of Theorem 1.3. First, we shall prove Theorem 1.3. Except for some sufficient modifications, the proof of the theorem follows the argument in [21. We denote by $\mathcal{D}$ the family of all dyadic cubes in $\mathbb{R}^{n}$. We assume that $f$ and $g$ are nonnegative, which may be done without any loss of generality thanks to the positivity of the integral kernel. We will denote by $B(x, r)$ the ball centered at $x$ and of radius $r$. We begin by discretizing the operator $T_{\rho} f$ following the idea of C. Pérez (see [17]):

$$
\begin{aligned}
T_{\rho} f(x) & =\sum_{\nu \in \mathbb{Z}} \int_{2^{\nu-1}<|x-y| \leq 2^{\nu}} f(y) \frac{\rho(|x-y|)}{|x-y|^{n}} d y \\
& \leq C \sum_{\nu \in \mathbb{Z}} \frac{\rho\left(2^{\nu}\right)}{2^{n \nu}} \int_{B\left(x, 2^{\nu}\right)} f(y) d y \\
& \leq C \sum_{\nu \in \mathbb{Z}} \sum_{Q \in \mathcal{D}: Q \ni x, \ell(Q)=2^{\nu}} \frac{\rho(\ell(Q))}{|Q|} \int_{3 Q} f(y) d y \\
& =C \sum_{Q \in \mathcal{D}} \frac{\rho(\ell(Q))}{|Q|} \int_{3 Q} f(y) d y \cdot \chi_{Q}(x) \\
& =C \sum_{Q \in \mathcal{D}} \rho(\ell(Q)) m_{3 Q}(f) \cdot \chi_{Q}(x),
\end{aligned}
$$


where we have used the doubling condition of $\rho$ for the first inequality. To prove Theorem 1.3, thanks to the doubling condition of $\tilde{\rho}^{a}$, which holds by use of the fact that $\tilde{\rho}(t)^{a}$ is nondecreasing and $\tilde{\rho}(t)^{a p} t^{-n}$ is nonincreasing, it suffices to show that

$$
\left(\int_{Q_{0}}\left(g(x) T_{\rho} f(x)\right)^{p} d x\right)^{1 / p} \leq C\|g\|_{q, \tilde{\rho}^{b}}\left\|M_{\tilde{\rho}^{1-b}} f\right\|_{p, \tilde{\rho}^{a}}\left|Q_{0}\right|^{1 / p} \tilde{\rho}\left(\ell\left(Q_{0}\right)\right)^{-a},
$$

for all dyadic cubes $Q_{0}$. Hereafter, we let

$$
\left\{\begin{array}{l}
\mathcal{D}_{1}\left(Q_{0}\right)=\left\{Q \in \mathcal{D}: Q \subset Q_{0}\right\} \\
\mathcal{D}_{2}\left(Q_{0}\right)=\left\{Q \in \mathcal{D}: Q \supsetneq Q_{0}\right\}
\end{array}\right.
$$

Let us define for $i=1,2$

$$
F_{i}(x)=\sum_{Q \in \mathcal{D}_{i}\left(Q_{0}\right)} \rho(\ell(Q)) m_{3 Q}(f) \chi_{Q}(x),
$$

and we shall estimate

$$
\left(\int_{Q_{0}}\left(g(x) F_{i}(x)\right)^{p} d x\right)^{1 / p}
$$

The case $i=1$ and $p=1$. We need the following crucial lemma, the proof of which is straightforward and is omitted (see [17, 21]).

Lemma 2.1. For a nonnegative function $h$ in $L^{\infty}\left(Q_{0}\right)$, we let $\gamma_{0}=m_{Q_{0}}(h)$ and $c=2^{n+1}$. For $k=1,2, \ldots$ let

$$
D_{k}=\bigcup_{Q \in \mathcal{D}_{1}\left(Q_{0}\right): m_{Q}(h)>\gamma_{0} c^{k}} Q .
$$

Considering the maximal cubes with respect to inclusion, we can write

$$
D_{k}=\bigcup_{j} Q_{k, j},
$$

where the cubes $\left\{Q_{k, j}\right\} \subset \mathcal{D}_{1}\left(Q_{0}\right)$ are nonoverlapping. By virtue of the maximality of $Q_{k, j}$ one has that

$$
\gamma_{0} c^{k}<m_{Q_{k, j}}(h) \leq 2^{n} \gamma_{0} c^{k}
$$

Let

$$
E_{0}=Q_{0} \backslash D_{1} \text { and } E_{k, j}=Q_{k, j} \backslash D_{k+1} .
$$

Then $\left\{E_{0}\right\} \cup\left\{E_{k, j}\right\}$ is a disjoint family of sets which decomposes $Q_{0}$ and satisfies

$$
\left|Q_{0}\right| \leq 2\left|E_{0}\right| \text { and }\left|Q_{k, j}\right| \leq 2\left|E_{k, j}\right|
$$

Also, we set

$$
\begin{aligned}
\mathcal{D}_{0} & =\left\{Q \in \mathcal{D}_{1}\left(Q_{0}\right): m_{Q}(h) \leq \gamma_{0} c\right\}, \\
\mathcal{D}_{k, j} & =\left\{Q \in \mathcal{D}_{1}\left(Q_{0}\right): Q \subset Q_{k, j}, \gamma_{0} c^{k}<m_{Q}(h) \leq \gamma_{0} c^{k+1}\right\} .
\end{aligned}
$$

Then

$$
\mathcal{D}_{1}\left(Q_{0}\right)=\mathcal{D}_{0} \cup \bigcup_{k, j} \mathcal{D}_{k, j}
$$


We need only verify that

$$
\int_{Q_{0}} g(x) F_{1}(x) d x \leq C\|g\|_{q, \tilde{\rho}^{b}} \int_{Q_{0}} M_{\tilde{\rho}^{1-b}} f(x) d x .
$$

Inserting the definition of $F_{1}$, we have

$$
\int_{Q_{0}} g(x) F_{1}(x) d x=\sum_{Q \in \mathcal{D}_{1}\left(Q_{0}\right)} \rho(\ell(Q)) m_{3 Q}(f) \int_{Q} g(x) d x
$$

Letting $h=g$, we shall apply Lemma 2.1 to the estimate of this quantity. Retaining the same notation as in Lemma 2.1 and noticing (2.2), we have

$$
\begin{aligned}
& \int_{Q_{0}} g(x) F_{1}(x) d x \\
& \quad=\sum_{Q \in \mathcal{D}_{0}} \rho(\ell(Q)) m_{3 Q}(f) \int_{Q} g(x) d x+\sum_{k, j} \sum_{Q \in \mathcal{D}_{k, j}} \rho(\ell(Q)) m_{3 Q}(f) \int_{Q} g(x) d x .
\end{aligned}
$$

We first evaluate

$$
\sum_{Q \in \mathcal{D}_{k, j}} \rho(\ell(Q)) m_{3 Q}(f) \int_{Q} g(x) d x
$$

It follows from the definition of $\mathcal{D}_{k, j}$ that (2.4) is bounded by

$$
C \gamma_{0} c^{k+1} \sum_{Q \in \mathcal{D}_{k, j}} \rho(\ell(Q)) \int_{3 Q} f(y) d y .
$$

The support condition and (1.7) gives us that

$$
\begin{aligned}
\sum_{Q \in \mathcal{D}_{k, j}} \rho(\ell(Q)) \int_{3 Q} f(y) d y & =\sum_{\nu=-\infty}^{\log _{2} \ell\left(Q_{k, j}\right)} \rho\left(2^{\nu}\right)\left(\sum_{Q \in \mathcal{D}_{k, j}: \ell(Q)=2^{\nu}} \int_{3 Q} f(y) d y\right) \\
& \leq C \int_{3 Q_{k, j}} f(y) d y\left(\sum_{\nu=-\infty}^{\log _{2} \ell\left(Q_{k, j}\right)} \rho\left(2^{\nu}\right)\right) \\
& \leq C \int_{3 Q_{k, j}} f(y) d y\left(\int_{0}^{\ell\left(Q_{k, j}\right)} \frac{\rho(s)}{s} d s\right) \\
& =C \tilde{\rho}\left(\ell\left(Q_{k, j}\right)\right) \int_{3 Q_{k, j}} f(y) d y .
\end{aligned}
$$

If we invoke relations $\left|Q_{k, j}\right| \leq 2\left|E_{k, j}\right|$ and $\gamma_{0} c^{k}<m_{Q_{k, j}}(g)$, then (2.4) is bounded by

$$
C \tilde{\rho}\left(\ell\left(Q_{k, j}\right)\right) m_{3 Q_{k, j}}(f) m_{Q_{k, j}}(g)\left|E_{k, j}\right| \cdot
$$

Now we have from the definition of the Morrey norm that

$$
m_{Q_{k, j}}(g) \leq m_{Q_{k, j}}^{(q)}(g) \leq\|g\|_{q, \tilde{\rho}} \tilde{\rho}\left(\ell\left(Q_{k, j}\right)\right)^{-b},
$$

and we conclude that

$$
(\underline{2.4}) \leq C\|g\|_{q, \tilde{\rho}^{b}} \tilde{\rho}\left(\ell\left(Q_{k, j}\right)\right)^{1-b} m_{3 Q_{k, j}}(f)\left|E_{k, j}\right| \leq C\|g\|_{q, \tilde{\rho}^{b}} \int_{E_{k, j}} M_{\tilde{\rho}^{1-b}} f(x) d x .
$$

Here, we have used the fact that $\tilde{\rho}$ is nondecreasing and

$$
\tilde{\rho}\left(\ell\left(3 Q_{k, j}\right)\right)^{1-b} m_{3 Q_{k, j}}(f) \leq \inf _{y \in Q_{k, j}} M_{\tilde{\rho}^{1-b}} f(y) .
$$


Similarly, we have

$$
\sum_{Q \in \mathcal{D}_{0}} \rho(\ell(Q)) m_{3 Q}(f) \int_{Q} g(x) d x \leq C\|g\|_{q, \tilde{\rho}^{b}} \int_{E_{0}} M_{\tilde{\rho}^{1-b}} f(x) d x .
$$

Summing up all factors, we obtain (2.3) by noticing $\left\{E_{0}\right\} \cup\left\{E_{k, j}\right\}$ is a disjoint family of sets which decomposes $Q_{0}$.

The case $i=1$ and $p>1$. In this case we establish

$$
\left(\int_{Q_{0}}\left(g(x) F_{1}(x)\right)^{p} d x\right)^{1 / p} \leq C\|g\|_{q, \tilde{\rho}^{b}}\left(\int_{Q_{0}} M_{\tilde{\rho}^{1-b}} f(x)^{p} d x\right)^{1 / p}
$$

by the duality argument. Take a nonnegative function $w \in L^{p^{\prime}}\left(Q_{0}\right), 1 / p+1 / p^{\prime}=1$, satisfying that $\|w\|_{L^{p^{\prime}}\left(Q_{0}\right)}=1$ and that

$$
\left(\int_{Q_{0}}\left(g(x) F_{1}(x)\right)^{p} d x\right)^{1 / p}=\int_{Q_{0}} g(x) F_{1}(x) w(x) d x .
$$

Letting $h=g w$, we shall apply Lemma 2.1 to estimate this quantity. It follows that

$$
\begin{aligned}
(2.6) \int_{Q_{0}} g(x) F_{1}(x) w(x) d x= & \sum_{Q \in \mathcal{D}_{1}\left(Q_{0}\right)} \rho(\ell(Q)) m_{3 Q}(f) \int_{Q} g(x) w(x) d x \\
= & \sum_{Q \in \mathcal{D}_{0}} \rho(\ell(Q)) m_{3 Q}(f) \int_{Q} g(x) w(x) d x \\
& +\sum_{k, j} \sum_{Q \in \mathcal{D}_{k, j}} \rho(\ell(Q)) m_{3 Q}(f) \int_{Q} g(x) w(x) d x .
\end{aligned}
$$

First, we evaluate

$$
\sum_{Q \in \mathcal{D}_{k, j}} \rho(\ell(Q)) m_{3 Q}(f) \int_{Q} g(x) w(x) d x .
$$

It follows from the same argument as above that (2.7) is bounded by

$$
C \tilde{\rho}\left(\ell\left(Q_{k, j}\right)\right) m_{3 Q_{k, j}}(f) m_{Q_{k, j}}(g w)\left|E_{k, j}\right| .
$$

These yield

$$
\begin{aligned}
(2.8) & \leq C \tilde{\rho}\left(\ell\left(Q_{k, j}\right)\right)^{1-b} m_{3 Q_{k, j}}(f) \tilde{\rho}\left(\ell\left(Q_{k, j}\right)\right)^{b} m_{Q_{k, j}}(g \cdot w)\left|E_{k, j}\right| \\
& \leq C \int_{E_{k, j}} M_{\tilde{\rho}^{1-b}} f(x) M_{\tilde{\rho}^{b}}[g \cdot w](x) d x .
\end{aligned}
$$

Similarly, we have

$$
\sum_{Q \in \mathcal{D}_{0}} \rho(\ell(Q)) m_{3 Q}(f) \int_{Q} g(x) w(x) d x \leq C\|g\|_{q, \tilde{\rho}^{b}} \int_{E_{0}} M_{\tilde{\rho}^{1-b}} f(x) M_{\tilde{\rho}^{b}}[g \cdot w](x) d x .
$$

Summing up all factors we obtain

$$
(\underline{2.6}) \leq C \int_{Q_{0}} M_{\tilde{\rho}^{1-b}} f(x) M_{\tilde{\rho}^{b}}[g \cdot w](x) d x .
$$

Another application of Hölder's inequality gives us that

$$
\text { (2.6) } \leq C\left(\int_{Q_{0}} M_{\tilde{\rho}^{1-b}} f(x)^{p} d x\right)^{1 / p}\left(\int_{Q_{0}} M_{\tilde{\rho}^{b}}[g \cdot w](x)^{p^{\prime}} d x\right)^{1 / p^{\prime}} \text {. }
$$


The fact $p^{\prime}>q^{\prime}$ and the $L^{p^{\prime} / q^{\prime}}$-boundedness of the maximal operator $M$ yield

$$
\begin{aligned}
(2.6) & \leq C\|g\|_{q, \tilde{\rho}^{b}}\left(\int_{Q_{0}} M_{\tilde{\rho}^{1-b}} f(x)^{p} d x\right)^{1 / p}\left(\int_{Q_{0}} w(x)^{p^{\prime}} d x\right)^{1 / p^{\prime}} \\
& =C\|g\|_{q, \tilde{\rho}^{b}}\left(\int_{Q_{0}} M_{\tilde{\rho}^{1-b}} f(x)^{p} d x\right)^{1 / p} .
\end{aligned}
$$

This is our desired inequality.

The case $i=2$ and $p \geq 1$. By a property of the dyadic cubes, for all $x \in Q_{0}$ we have

$$
F_{2}(x)=\sum_{Q \in \mathcal{D}_{2}\left(Q_{0}\right)} \rho(\ell(Q)) m_{3 Q}(f)
$$

and

$$
\begin{aligned}
\rho(\ell(Q)) m_{3 Q}(f) & =\rho(\ell(Q)) \tilde{\rho}(\ell(Q))^{b-1} \cdot \tilde{\rho}(\ell(Q))^{1-b} m_{3 Q}(f) \\
& \leq \rho(\ell(Q)) \tilde{\rho}(\ell(Q))^{b-1} m_{Q}\left(M_{\tilde{\rho}^{1-b}} f\right) .
\end{aligned}
$$

It follows that

$$
m_{Q}\left(M_{\tilde{\rho}^{1-b}} f\right) \leq m_{Q}^{(p)}\left(M_{\tilde{\rho}^{1-b}} f\right) \leq\left\|M_{\tilde{\rho}^{1-b}} f\right\|_{p, \tilde{\rho}^{a}} \tilde{\rho}(\ell(Q))^{-a} .
$$

Inserting these estimates, we obtain

$$
\begin{aligned}
F_{2}(x) & \leq C\left\|M_{\tilde{\rho}^{1-b}} f\right\|_{p, \tilde{\rho}^{a}} \sum_{Q \in \mathcal{D}_{2}\left(Q_{0}\right)} \rho(\ell(Q)) \tilde{\rho}(\ell(Q))^{b-a-1} \\
& \leq C\left\|M_{\tilde{\rho}^{1-b}} f\right\|_{p, \tilde{\rho}^{a}} \sum_{\nu=1+\log _{2} \ell\left(Q_{0}\right)}^{\infty} \rho\left(2^{\nu}\right) \tilde{\rho}\left(2^{\nu}\right)^{b-a-1} \\
& \leq C\left\|M_{\tilde{\rho}^{1-b}} f\right\|_{p, \tilde{\rho}^{a}} \int_{\ell\left(Q_{0}\right)}^{\infty} \tilde{\rho}(s)^{b-a-1} \frac{\rho(s)}{s} d s .
\end{aligned}
$$

Here, in the last inequality we have used (1.7) and the fact that $\tilde{\rho}$ is nondecreasing. Now we see that

$$
\int_{\ell\left(Q_{0}\right)}^{\infty} \tilde{\rho}(s)^{b-a-1} \frac{\rho(s)}{s} d s=\int_{\ell\left(Q_{0}\right)}^{\infty} \frac{d}{d s}\left(\frac{1}{b-a} \tilde{\rho}(s)^{b-a}\right) d s \leq \frac{\tilde{\rho}\left(\ell\left(Q_{0}\right)\right)^{b-a}}{a-b},
$$

where we have used $b<a$ for the last inequality. Thus, for all $x \in Q_{0}$ we obtain

$$
F_{2}(x) \leq C\left\|M_{\tilde{\rho}^{1-b}} f\right\|_{p, \tilde{\rho}^{a}} \tilde{\rho}\left(\ell\left(Q_{0}\right)\right)^{b-a}
$$

and

$$
\begin{aligned}
\left(\int_{Q_{0}}\left(g(x) F_{2}(x)\right)^{p} d x\right)^{1 / p} & \leq C m_{Q_{0}}^{(p)}(g)\left\|M_{\tilde{\rho}^{1-b}} f\right\|_{p, \tilde{\rho}^{a}} \tilde{\rho}\left(\ell\left(Q_{0}\right)\right)^{b-a}\left|Q_{0}\right|^{1 / p} \\
& \leq C \tilde{\rho}\left(\ell\left(Q_{0}\right)\right)^{b-a} m_{Q_{0}}^{(q)}(g)\left\|M_{\tilde{\rho}^{1-b}} f\right\|_{p, \tilde{\rho}^{a}}\left|Q_{0}\right|^{1 / p} \\
& \leq C\|g\|_{q, \tilde{\rho}^{b}}\left\|M_{\tilde{\rho}^{1-b}} f\right\|_{p, \tilde{\rho}^{a}} \tilde{\rho}\left(\ell\left(Q_{0}\right)\right)^{-a}\left|Q_{0}\right|^{1 / p} .
\end{aligned}
$$

This is our desired inequality.

Remark 2.2. Let $1 \leq p<\infty$. Suppose that $\phi$ satisfies (1.2). In the course of the proof, if we let $b=0$ and $g \equiv 1$, then we have

$$
\left(\int_{Q_{0}} F_{1}(x)^{p} d x\right)^{1 / p} \leq C\left(\int_{Q_{0}} M_{\tilde{\rho}} f(x)^{p} d x\right)^{1 / p} .
$$


Also, if we mimic the way to obtain (2.9), then for all $x \in Q_{0}$ we have

$$
F_{2}(x) \leq C\left\|M_{\tilde{\rho}} f\right\|_{p, \phi} \int_{\ell\left(Q_{0}\right)}^{\infty} \frac{\rho(s)}{s \tilde{\rho}(s) \phi(s)} d s,
$$

and hence

$$
\left(\int_{Q_{0}} F_{2}(x)^{p} d x\right)^{1 / p} \leq C\left\|M_{\tilde{\rho}} f\right\|_{p, \phi}\left|Q_{0}\right|^{1 / p} \int_{\ell\left(Q_{0}\right)}^{\infty} \frac{\rho(s)}{s \tilde{\rho}(s) \phi(s)} d s .
$$

These imply

$$
\left\|T_{\rho} f\right\|_{p, \phi} \leq C\left\|M_{\tilde{\rho}} f\right\|_{p, \phi}
$$

if, for all $t \geq 0, \phi$ satisfies further that

$$
\phi(t) \int_{t}^{\infty} \frac{\rho(s)}{s \tilde{\rho}(s) \phi(s)} d s \leq C .
$$

Remark 2.3. Let $1 \leq p, q<\infty$. Suppose that $\phi$ satisfies (1.2). Then, similarly, we see that

$$
C\left(\int_{Q_{0}} T_{\rho} f(x)^{q} d x\right)^{1 / q} \leq\left(\int_{Q_{0}} M_{\tilde{\rho}} f(x)^{q} d x\right)^{1 / q}+\left|Q_{0}\right|^{1 / q}\|f\|_{p, \phi} \int_{\ell\left(Q_{0}\right)}^{\infty} \frac{\rho(s)}{s \phi(s)} d s .
$$

2.2. Proof of Theorem 1.1. We need some lemmas.

Lemma 2.4. Let $p>1$. Suppose that $\phi$ satisfies (1.2). Then

$$
\|M f\|_{p, \phi} \leq C\|f\|_{p, \phi} .
$$

Proof. Fix a cube $Q_{0}$. Let $f_{1}=\chi_{3 Q_{0}} f$ and $f_{2}=f-f_{1}$. Then $M f(x) \leq M f_{1}(x)+$ $M f_{2}(x)$. It follows from the definition of $M$ that for all $x \in Q_{0}$,

$$
M f_{2}(x)=\sup _{x \in Q \in \mathcal{Q}: \ell(Q) \geq \ell\left(Q_{0}\right)} \frac{1}{|Q|} \int_{Q}|f(y)| d y .
$$

Suppose that $x \in Q_{0}, x \in Q \in \mathcal{Q}$ and $\ell(Q) \geq \ell\left(Q_{0}\right)$. Then

$$
\phi\left(\ell\left(Q_{0}\right)\right) m_{Q}(|f|) \leq \phi(\ell(Q)) m_{Q}^{(p)}(|f|) \leq\|f\|_{p, \phi},
$$

where we have used Hölder's inequality and the fact that $\phi$ is nondecreasing.

This gives us that

$$
\phi\left(\ell\left(Q_{0}\right)\right) M f_{2}(x) \leq\|f\|_{p, \phi} \text { for all } x \in Q_{0}
$$

and that

$$
\begin{aligned}
\phi\left(\ell\left(Q_{0}\right)\right) m_{Q_{0}}^{(p)}(M f)^{p} & \leq \phi\left(\ell\left(Q_{0}\right)\right) m_{Q_{0}}^{(p)}\left(M f_{1}\right)^{p}+\phi\left(\ell\left(Q_{0}\right)\right) m_{Q_{0}}^{(p)}\left(M f_{2}\right)^{p} \\
& \leq C \phi\left(\ell\left(3 Q_{0}\right)\right) m_{3 Q_{0}}^{(p)}(f)+\|f\|_{p, \phi} \leq C\|f\|_{p, \phi},
\end{aligned}
$$

where we have used $L^{p}$ boundedness of the maximal operator $M$. This implies our desired inequality.

Remark 2.5. A similar proof can be found in [18, Theorem 2.3].

Lemma 2.6. Let $1<p \leq q<\infty$. Suppose that $\phi$ satisfies (1.2). Then

$$
\left\|M_{\phi^{1-p / q}} f\right\|_{q, \phi^{p / q}} \leq C\|f\|_{p, \phi} \text {. }
$$


Proof. Let $x \in \mathbb{R}^{n}$ be a fixed point. For every cube $Q \ni x$ we see that

$$
\begin{aligned}
\phi(\ell(Q))^{1-p / q} m_{Q}(|f|) & \leq \min \left(\phi(\ell(Q))^{1-p / q} M f(x), \phi(\ell(Q))^{-p / q}\|f\|_{p, \phi}\right) \\
& \leq \sup _{t \geq 0} \min \left(t^{1-p / q} M f(x), t^{-p / q}\|f\|_{p, \phi}\right) \\
& =\|f\|_{p, \phi}^{1-p / q} M f(x)^{p / q} .
\end{aligned}
$$

This implies

$$
M_{\phi^{1-p / q}} f(x)^{q} \leq\|f\|_{p, \phi}^{q-p} M f(x)^{p} .
$$

It follows from Lemma 2.4 that for every cube $Q_{0}$,

$$
m_{Q_{0}}^{(q)}\left(M_{\phi^{1-p / q}} f\right) \leq\|f\|_{p, \phi}^{1-p / q} m_{Q_{0}}^{(p)}(M f)^{p / q} \leq C\|f\|_{p, \phi} \phi\left(\ell\left(Q_{0}\right)\right)^{-p / q} .
$$

The desired inequality then follows.

For $b=1$ Theorem 1.1 can be proved by using Corollary 1.5 and Lemma 2.4. and for $b<1$ it can be proved by using the following:

Lemma 2.7. Let $1<p<\infty, a>1$ and $(a-1) q=a p$. Assume that $\rho(t)$ is nondecreasing and $\rho(t)^{a p} t^{-n}$ is nonincreasing. Then

$$
\left\|M_{\rho} f\right\|_{q, \rho^{a-1}} \leq C\|f\|_{p, \rho^{a}} .
$$

Proof. To prove this lemma we merely check all the conditions of Lemma 2.6 when $\phi=\rho^{a}$.

Remark 2.8. Let $1<p \leq q<\infty$. Suppose that $\phi$ satisfies (1.2). Remark 2.3 and Lemma 2.6 give us that

$$
\left\|T_{\rho} f\right\|_{q, \phi^{p / q}} \leq C\|f\|_{p, \phi}
$$

if, for all $t \geq 0, \phi$ satisfies further that

$$
\phi(t)^{p / q} \int_{t}^{\infty} \frac{\rho(s)}{s \phi(s)} d s \leq C \text { and } \tilde{\rho}(t) \leq C \phi(t)^{1-p / q} .
$$

This condition can be restated as

$$
\frac{\tilde{\rho}(t)}{\phi(t)}+\int_{t}^{\infty} \frac{\rho(s)}{s \phi(s)} d s \leq C \phi(t)^{-p / q}, \quad t \geq 0,
$$

and can be seen in [ 8 , Theorem 3.1] with some superfluous conditions.

2.3. Proof of Theorem 1.7. Since the operator $M_{\rho}$ is nonlinear, an alternative approach will be necessary. First of all, we notice that $\rho$ satisfies the doubling condition (with the doubling constant $2^{a p}$ ), which can be proved by using the conditions postulated on $\rho$. The monotonicity of $\rho$ yields

$$
\frac{1}{C_{2}} M_{\rho} f(x) \leq M_{d ; \rho} f(x) \leq C_{2} M_{\rho} f(x) \text { for a.e. } x \in \mathbb{R}^{n},
$$

where

$$
M_{d ; \rho} f(x)=\sup _{x \in Q \in \mathcal{D}} \rho(\ell(Q)) m_{3 Q}(f) .
$$

Thanks to the doubling condition of $\rho$, to prove Theorem 1.7 it suffices to show that

$$
\left(\int_{Q_{0}}\left(g(x) M_{d ; \rho} f(x)\right)^{p} d x\right)^{1 / p} \leq C\|g\|_{p, \rho^{b}}\left\|M_{d ; \rho^{1-b}} f\right\|_{p, \rho^{a}}\left|Q_{0}\right|^{1 / p} \rho\left(\ell\left(Q_{0}\right)\right)^{-a}
$$


for all dyadic cubes $Q_{0}$. Fix $Q_{0} \in \mathcal{D}$ and recall $\mathcal{D}_{1}\left(Q_{0}\right), \mathcal{D}_{2}\left(Q_{0}\right)$ defined earlier. Then it follows that

$$
M_{d ; \rho} f(x) \leq \hat{M}_{d ; \rho} f(x)+\check{M}_{d ; \rho} f(x),
$$

provided that

$$
\left\{\begin{array}{l}
\hat{M}_{d ; \rho} f(x)=\sup _{x \in Q \in \mathcal{D}_{1}\left(Q_{0}\right)} \rho(\ell(Q)) m_{3 Q}(f), \\
\check{M}_{d ; \rho} f(x)=\sup _{x \in Q \in \mathcal{D}_{2}\left(Q_{0}\right)} \rho(\ell(Q)) m_{3 Q}(f) .
\end{array}\right.
$$

If $Q \in \mathcal{D}_{2}\left(Q_{0}\right)$, then

$$
\begin{aligned}
\rho(\ell(Q)) m_{3 Q}(f) & =\rho(\ell(Q))^{b} m_{Q}^{(p)}\left(\rho(\ell(Q))^{1-b} m_{3 Q}(f)\right) \\
& \leq \rho(\ell(Q))^{b-a} \rho(\ell(Q))^{a} m_{Q}^{(p)}\left(M_{d ; \rho^{1-b}} f\right) \\
& \leq \rho\left(\ell\left(Q_{0}\right)\right)^{b-a}\left\|M_{d ; \rho^{1-b}} f\right\|_{p, \rho^{a}} .
\end{aligned}
$$

Here, we have used $Q_{0} \subset Q$ and $b \leq a$. For all $x \in Q_{0}$ this implies that

$$
\check{M}_{d ; \rho} f(x) \leq \rho\left(\ell\left(Q_{0}\right)\right)^{b-a}\left\|M_{d ; \rho^{1-b}} f\right\|_{p, \rho^{a}},
$$

and hence

$$
\rho\left(\ell\left(Q_{0}\right)\right)^{a} m_{Q_{0}}^{(p)}\left(g \check{M}_{d ; \rho} f\right) \leq\|g\|_{p, \rho^{b}}\left\|M_{d ; \rho^{1-b}} f\right\|_{p, \rho^{a}} .
$$

Thus, we concentrate ourselves on estimating the integral

$$
\int_{Q_{0}}\left(g(x) \hat{M}_{d ; \rho} f(x)\right)^{p} d x .
$$

A standard density argument allows us to assume that $f$ is bounded. One knows that for each $\lambda>0$ there exists a nonoverlapping collection of maximal (with respect to inclusion) dyadic cubes $\left\{Q_{i}^{\lambda}\right\} \subset \mathcal{D}_{1}\left(Q_{0}\right)$ such that

$$
\left\{x \in Q_{0}: \hat{M}_{d ; \rho} f(x)>\lambda\right\}=\bigcup_{i} Q_{i}^{\lambda} .
$$

It follows from the definitions that

$$
\rho\left(\ell\left(Q_{i}^{\lambda}\right)\right) \geq \frac{\rho\left(\ell\left(Q_{0}\right)\right) m_{3 Q_{0}}(f)}{\|f\|_{L^{\infty}\left(3 Q_{0}\right)}}
$$

for every $\lambda>0$ and $i$.

In view of the distribution function of $\hat{M}_{d ; \rho} f$, we can rewrite the integral as

$$
p \int_{0}^{\infty} \sum_{i} g^{p}\left(Q_{i}^{\lambda}\right) \lambda^{p-1} d \lambda
$$

where $g^{p}(Q)=\int_{Q} g(x)^{p} d x$. For every $Q \in\left\{Q_{i}^{\lambda}\right\}_{\lambda>0} \backslash\left\{Q_{0}\right\}$ define the mother cube $\mu(Q)$ by a minimal cube $Q^{\prime}$ such that $Q \subset Q^{\prime} \in\left\{Q_{i}^{\lambda}\right\}$ and $\ell(Q)<\ell\left(Q^{\prime}\right)$. Then we can rewrite the integral further (assuming that $\mu\left(Q_{0}\right)$ is an empty set and the integral over $\mu\left(Q_{0}\right)$ is understood as zero) as

$$
\sum_{Q \in\left\{Q_{i}^{\lambda}\right\}} g^{p}(Q)\left(\left(\rho(\ell(Q)) m_{3 Q}(f)\right)^{p}-\left(\rho(\ell(\mu(Q))) m_{3 \mu(Q)}(f)\right)^{p}\right) .
$$


By use of $g^{p}(Q) \leq\|g\|_{p, \rho^{b}} p|Q| \rho(\ell(Q))^{-b p}$ and $\rho(\ell(Q)) \leq \rho(\ell(\mu(Q)))$, this term is bounded by a $\|g\|_{p, \rho^{b}}{ }^{p}$ multiple of the following:

$$
\begin{aligned}
\sum_{Q \in\left\{Q_{i}^{\lambda}\right\}}|Q|\left(\left(\frac{\rho(\ell(Q))}{\rho(\ell(Q))^{b}} m_{3 Q}(f)\right)^{p}-\left(\frac{\rho(\ell(\mu(Q)))}{\rho(\ell(Q))^{b}} m_{3 \mu(Q)}(f)\right)^{p}\right) \\
\leq \sum_{Q \in\left\{Q_{i}^{\lambda}\right\}}|Q|\left(\left(\rho(\ell(Q))^{1-b} m_{3 Q}(f)\right)^{p}-\left(\rho(\ell(\mu(Q)))^{1-b} m_{3 \mu(Q)}(f)\right)^{p}\right) .
\end{aligned}
$$

For $x \in Q_{0}$ define the cube $Q_{x}$ by a minimal cube $Q$ such that $x \in Q \in\left\{Q_{i}^{\lambda}\right\}$, and define an operator $S$ by

$$
S f(x)=\rho\left(\ell\left(Q_{x}\right)\right)^{1-b} m_{3 Q_{x}}(f) .
$$

Then we assert that (2.10) is equal to $\int_{Q_{0}} S f(x)^{p} d x$. In fact, by Fubini's theorem

$$
\begin{aligned}
& \sum_{Q \in\left\{Q_{i}^{\lambda}\right\}}|Q|\left(\left(\rho(\ell(Q))^{1-b} m_{3 Q}(f)\right)^{p}-\left(\rho(\ell(\mu(Q)))^{1-b} m_{3 \mu(Q)}(f)\right)^{p}\right) \\
& =\int_{Q_{0}} \sum_{Q \in\left\{Q_{i}^{\lambda}\right\}}\left(\left(\rho(\ell(Q))^{1-b} m_{3 Q}(f)\right)^{p}-\left(\rho(\ell(\mu(Q)))^{1-b} m_{3 \mu(Q)}(f)\right)^{p}\right) \chi_{Q}(x) d x \\
& =\int_{Q_{0}}\left(\rho\left(\ell\left(Q_{x}\right)\right)^{1-b} m_{3 Q_{x}}(f)\right)^{p} d x=\int_{Q_{0}} S f(x)^{p} d x .
\end{aligned}
$$

Clearly, from $S f(x) \leq M_{d ; \rho^{1-b}} f(x)$, we obtain

$$
\left(\int_{Q_{0}}\left(g(x) \hat{M}_{d ; \rho} f(x)\right)^{p} d x\right)^{1 / p} \leq\|g\|_{p, \rho^{b}}\left(\int_{Q_{0}} M_{d ; \rho^{1-b}} f(x)^{p} d x\right)^{1 / p} .
$$

This is our desired inequality.

\section{A dual Version of Olsen's inequality}

In this section, as an application of Theorem 1.1 we consider a dual version of Olsen's inequality on the predual of a Morrey space (Theorem 3.1). As a corollary (Corollary 3.2), we have the boundedness properties of the operator $T_{\rho}$ on the predual of a Morrey space. We shall define the block spaces following [16].

Let $1<p<\infty$ and $1 / p+1 / p^{\prime}=1$. Suppose that $\phi$ satisfies (1.2). We say that a function $b$ on $\mathbb{R}^{n}$ is a $\left(p^{\prime}, \phi\right)$-block provided that $b$ is supported on a cube $Q \subset \mathbb{R}^{n}$ and satisfies

$$
m_{Q}^{\left(p^{\prime}\right)}(b) \leq \frac{\phi(\ell(Q))}{|Q|} .
$$

If, in addition, $b$ is a continuous function, then we say that $b$ is a continuous $\left(p^{\prime}, \phi\right)$ block. The space $\mathcal{B}^{p^{\prime}, \phi}\left(\mathbb{R}^{n}\right)=\mathcal{B}^{p^{\prime}, \phi}$ is defined by the set of all functions $f$ locally in $L^{p^{\prime}}\left(\mathbb{R}^{n}\right)$ with the norm

$$
\|f\|_{\mathcal{B}^{p^{\prime}, \phi}}=\inf \left\{\left\|\left\{\lambda_{k}\right\}\right\|_{l^{1}}: f=\sum_{k} \lambda_{k} b_{k}\right\}<\infty,
$$

where $b_{k}$ is a $\left(p^{\prime}, \phi\right)$-block and $\left\|\left\{\lambda_{k}\right\}\right\|_{l^{1}}=\sum_{k}\left|\lambda_{k}\right|<\infty$, and the infimum is taken over all possible decompositions of $f$. If $\phi(t) \equiv t^{n / p_{0}}, p_{0} \geq p, \mathcal{B}^{p^{\prime}, \phi}$ is the usual block 
space, which we write for $\mathcal{B}^{p^{\prime}, p_{0}^{\prime}}$ and the norm for $\|\cdot\|_{\mathcal{B}^{p^{\prime}, p_{0}^{\prime}}}$, because the right-hand side of (3.1) is equal to $|Q|^{1 / p_{0}-1}=|Q|^{-1 / p_{0}^{\prime}}$. Then we have the inclusion

$$
L^{p_{0}^{\prime}}=\mathcal{B}^{p_{0}^{\prime}, p_{0}^{\prime}} \supset \mathcal{B}^{p_{1}^{\prime}, p_{0}^{\prime}} \supset \mathcal{B}^{p_{2}^{\prime}, p_{0}^{\prime}}
$$

when $1<p_{0}^{\prime} \leq p_{1}^{\prime} \leq p_{2}^{\prime}<\infty$. In [16, Theorem 1] or [22, Proposition 5], the following is essentially proved:

The predual space of $\mathcal{M}^{p, \phi}$ is $\mathcal{B}^{p^{\prime}, \phi}$ in the following sense: If $g \in$ $\mathcal{M}^{p, \phi}$, then $\int_{\mathbb{R}^{n}} f(x) g(x) d x$ is an element of $\left(\mathcal{B}^{p^{\prime}, \phi}\right)^{*}$. Moreover, for any $L \in\left(\mathcal{B}^{p^{\prime}, \phi}\right)^{*}$, there exists $g \in \mathcal{M}^{p, \phi}$ such that

$$
L(f)=\int_{\mathbb{R}^{n}} f(x) g(x) d x, \quad f \in \mathcal{B}^{p^{\prime}, \phi} .
$$

In this section we shall prove the following theorem.

Theorem 3.1. Let $1<p<\infty, q>r, 0 \leq b \leq 1, a>1$ and $(a+b-1) r=a p$. Suppose that $\rho$ satisfies (1.5), (1.6) and that $\tilde{\rho}(t)^{\max (a p, b q)} t^{-n}$ is nonincreasing. Then

$$
\left\|T_{\rho}(g f)\right\|_{\mathcal{B}^{p^{\prime}, \tilde{\rho}^{a}}} \leq C\|g\|_{\mathcal{M}^{q, \tilde{\rho}^{b}}}\|f\|_{\mathcal{B}^{r^{\prime}, \tilde{\rho}^{a+b-1}}}
$$

if $g$ is a continuous function.

Corollary 3.2. Let $1<p<\infty, a>1$ and $(a-1) r=a p$. Suppose that $\rho$ satisfies (1.5), (1.6) and that $\tilde{\rho}(t)^{a p} t^{-n}$ is nonincreasing. Then

$$
\left\|T_{\rho} f\right\|_{\mathcal{B}^{p^{\prime}, \tilde{\rho}^{a}}} \leq C\|f\|_{\mathcal{B}^{r^{\prime}, \tilde{\rho}^{a-1}}} .
$$

To prove the theorem we need some elementary lemmas.

Lemma 3.3. For any cube $Q$ and $f$ locally in $L^{p^{\prime}}\left(\mathbb{R}^{n}\right)$ we have

$$
\left\|\chi_{Q} f\right\|_{\mathcal{B}^{p^{\prime}, \phi}} \leq \frac{|Q|^{1 / p}}{\phi(\ell(Q))}\left\|\chi_{Q} f\right\|_{p^{\prime}}
$$

Proof. Just observe that

$$
\chi_{Q} f=\frac{|Q|^{1 / p}}{\phi(\ell(Q))}\left\|\chi_{Q} f\right\|_{p^{\prime}} b
$$

with $b$ a $\left(p^{\prime}, \phi\right)$-block.

Lemma 3.4. A function $f$ belongs to $\mathcal{B}^{p^{\prime}, \phi}$ if and only if there exists $g \in \mathcal{B}^{p^{\prime}, \phi}$ such that $|f(x)| \leq g(x)$ a.e. $x \in \mathbb{R}^{n}$.

Proof. Suppose that $f \in \mathcal{B}^{p^{\prime}, \phi}$. Then there exist a $\left(p^{\prime}, \phi\right)$-block $b_{k}$ and a sequence $\left\{\lambda_{k}\right\} \in l^{1}$ such that $f=\sum_{k} \lambda_{k} b_{k}$. Letting $g=\sum_{k}\left|\lambda_{k}\right|\left|b_{k}\right|$, we have $g \in \mathcal{B}^{p^{\prime}, \phi}$ and $|f| \leq g$. Conversely, suppose that there exists $g \in \mathcal{B}^{p^{\prime}, \phi}$ which satisfies $|f(x)| \leq$ $g(x)$. Decompose $g$ as $g=\sum_{k} \lambda_{k}^{\prime} b_{k}^{\prime}$, where $b_{k}^{\prime}$ is a $\left(p^{\prime}, \phi\right)$-block and $\left\{\lambda_{k}^{\prime}\right\} \in l^{1}$. Then we see that

$$
\chi_{\{y: g(y) \neq 0\}}(x)=\sum_{k} \lambda_{k}^{\prime} \frac{1}{g(x)} b_{k}^{\prime}(x),
$$

and hence

$$
f(x)=\sum_{k} \lambda_{k}^{\prime} \frac{f(x)}{g(x)} b_{k}^{\prime}(x)
$$


Since $\frac{|f(x)|}{g(x)} \leq 1$, the function $\frac{f(x)}{g(x)} b_{k}^{\prime}(x)$ becomes a $\left(p^{\prime}, \phi\right)$-block. This proves the lemma.

Lemma 3.5. Let $\tilde{\mathcal{B}}^{p^{\prime}, \phi}$ be the set of all functions $f$ in $\mathcal{B}^{p^{\prime}, \phi}$ which is generated by continuous $\left(p^{\prime}, \phi\right)$-blocks. Then $\tilde{\mathcal{B}}^{p^{\prime}, \phi}$ is a dense subspace of $\mathcal{B}^{p^{\prime}, \phi}$.

Proof. Suppose that $f \in \mathcal{B}^{p^{\prime}, \phi}$. Then $f$ admits a decomposition $f=\sum_{k} \lambda_{k} b_{k}$, where each $b_{k}$ is a $\left(p^{\prime}, \phi\right)$-block which is supported on a cube $Q_{k}$ and $\left\{\lambda_{k}\right\} \in l^{1}$. If $\varepsilon>0$ is given, choose a continuous function $c_{k}$ such that $\left|c_{k}(x)\right| \leq\left|b_{k}(x)\right|$ and

$$
\left\|b_{k}-c_{k}\right\|_{p^{\prime}} \leq \frac{\phi\left(\ell\left(Q_{k}\right)\right)}{\left|Q_{k}\right|^{1 / p}} \frac{\varepsilon}{\left\|\left\{\lambda_{k}\right\}\right\|_{l^{1}}} .
$$

Now, we let $g=\sum_{k} \lambda_{k} c_{k}$. Since $c_{k}$ is a continuous $\left(p^{\prime}, \phi\right)$-block, $g$ belongs to $\tilde{\mathcal{B}}^{p^{\prime}, \phi}$ and, by using Lemma 3.3. we see that

$$
\|f-g\|_{\mathcal{B}^{p^{\prime}, \phi}} \leq \sum_{k}\left|\lambda_{k}\right| \cdot\left\|b_{k}-c_{k}\right\|_{\mathcal{B}^{p^{\prime}, \phi}} \leq \varepsilon .
$$

Thus, the proof is complete.

Proof of Theorem 3.1. Fix a continuous function $g \in \mathcal{M}^{q, \tilde{\rho}^{b}}$ so that it satisfies $\|g\|_{\mathcal{M}^{q, \tilde{\rho}^{b}}}=1$. By Lemma 3.5 it suffices to show the continuity on the subspace $\tilde{\mathcal{B}}^{r^{\prime}, \tilde{\rho}^{a+b-1}}$. Moreover, thanks to the linearity, we may assume that $f$ is a continuous $\left(r^{\prime}, \tilde{\rho}^{a+b-1}\right)$-block. By the Hahn-Banach theorem, once we verify $T_{\rho}(g f) \in \mathcal{B}^{p^{\prime}, \tilde{\rho}^{a}}$, then

$$
\begin{aligned}
& \left\|T_{\rho}(g f)\right\|_{\mathcal{B}^{p^{\prime}, \tilde{\rho}^{a}}} \\
& \quad=\sup _{w}\left|\int_{\mathbb{R}^{n}} T_{\rho}(g f)(x) w(x) d x\right|=\sup _{w}\left|\int_{\mathbb{R}^{n}} g(x) T_{\rho} w(x) \cdot f(x) d x\right|,
\end{aligned}
$$

where the supremum is taken over all the normalized $w \in \mathcal{M}^{p, \tilde{\rho}^{a}}$, and, by use of Theorem [1.1, one would conclude that $\left\|T_{\rho}(g f)\right\|_{\mathcal{B}^{p^{\prime}, \tilde{\rho}^{a}}} \leq C$. Thus, we need only verify that $T_{\rho}(g f)$ belongs to $\mathcal{B}^{p^{\prime}, \tilde{\rho}^{a}}$. We may assume further without loss of generality that $f$ and $g$ are nonnegative and that $f$ is supported on a dyadic cube $Q_{0} \in \mathcal{D}$ and satisfies

$$
\left(\int_{Q_{0}} f(x)^{r^{\prime}} d x\right)^{1 / r^{\prime}} \leq \frac{\tilde{\rho}\left(\ell\left(Q_{0}\right)\right)^{a+b-1}}{\left|Q_{0}\right|^{1 / r}} .
$$

In the same manner as in the previous section, noting Lemma 3.4, we wish to estimate

$$
F_{i}(x)=\sum_{Q \in \mathcal{D}_{i}\left(Q_{0}\right)} \rho(\ell(Q)) m_{3 Q}(g f) \chi_{Q}(x), \quad i=1,2,
$$

in view of the decomposition into blocks.

The case $i=1$. It follows from (1.7) that

$$
\rho(\ell(Q)) m_{3 Q}(g f) \chi_{Q}(x) \leq C \tilde{\rho}(\ell(Q)) m_{3 Q}(g f) \chi_{Q}(x) .
$$


First, we assert that the right-hand side of (3.3) becomes a $\left(p^{\prime}, \tilde{\rho}^{a}\right)$-block if $Q \in$ $\mathcal{D}_{1}\left(Q_{0}\right)$. Indeed,

$$
\begin{aligned}
& \left(\int_{Q}\left(\tilde{\rho}(\ell(Q)) m_{3 Q}(g f) \chi_{Q}(x)\right)^{p^{\prime}} d x\right)^{1 / p^{\prime}} \\
& =\tilde{\rho}(\ell(Q)) m_{3 Q}(g f)|Q|^{1-1 / p} \leq \tilde{\rho}(\ell(Q))|Q|^{1-1 / p} m_{3 Q}^{(r)}(g) m_{3 Q}^{\left(r^{\prime}\right)}(f) \\
& \leq C \tilde{\rho}(\ell(Q))|Q|^{1 / r-1 / p} m_{3 Q}^{(q)}(g)\left(\int_{Q_{0}} f(y)^{r^{\prime}} d y\right)^{1 / r^{\prime}} \\
& \leq C \tilde{\rho}(\ell(Q))^{1-b}|Q|^{1 / r-1 / p} \frac{\tilde{\rho}\left(\ell\left(Q_{0}\right)\right)^{a+b-1}}{\left|Q_{0}\right|^{1 / r}} \leq C \frac{\tilde{\rho}(\ell(Q))^{a}}{|Q|^{1 / p}}
\end{aligned}
$$

where we have used (3.2) and the fact that $q>r, m_{3 Q}^{(q)}(g) \leq \tilde{\rho}(\ell(Q))^{-b}$ and $\tilde{\rho}(t)^{(a+b-1) r} t^{-n}$ is nonincreasing.

Let $\varepsilon=\tilde{\rho}\left(\ell\left(Q_{0}\right)\right)^{a-1}\left|Q_{0}\right|^{-n}$. Since $g f$ is uniformly continuous, we can choose $\delta>0$ such that $|g(x) f(x)-g(y) f(y)| \leq \varepsilon$ whenever $|x-y| \leq \delta$. Let $\mathcal{F}$ be the set of all dyadic cubes $Q \in \mathcal{D}_{1}\left(Q_{0}\right)$ such that $\ell(Q) \leq \frac{\delta}{3 \sqrt{n}}$, and let $\mathcal{F}_{0}$ be the set of all maximal (with respect to inclusion) cubes in $\mathcal{F}$. Take a cube $Q_{1} \in \mathcal{F}_{0}$. Then, for every dyadic cube $Q \subset Q_{1}$, we see that

$$
\begin{aligned}
m_{3 Q}(g f) & \leq \frac{1}{|3 Q|} \int_{3 Q}\left|g(y) f(y)-\min _{z \in 3 Q_{1}} g(z) f(z)\right| d y+\min _{z \in 3 Q_{1}} g(z) f(z) \\
& \leq \varepsilon+m_{3 Q_{1}}(g f) .
\end{aligned}
$$

This gives us that

$$
\begin{aligned}
& \sum_{Q \in \mathcal{D}_{1}\left(Q_{0}\right): Q \subset Q_{1}} \rho(\ell(Q)) m_{3 Q}(g f) \chi_{Q}(x) \\
\leq & \left(\varepsilon+m_{3 Q_{1}}(g f)\right)\left(\sum_{\nu=-\infty}^{\log _{2} \ell\left(Q_{1}\right)} \rho\left(2^{\nu}\right)\right) \chi_{Q_{1}}(x) \\
\leq & C\left(\varepsilon \tilde{\rho}\left(\ell\left(Q_{1}\right)\right) \chi_{Q_{1}}(x)+\tilde{\rho}\left(\ell\left(Q_{1}\right)\right) m_{3 Q_{1}}(g f) \chi_{Q_{1}}(x)\right) .
\end{aligned}
$$

These yield

$$
\begin{aligned}
& \sum_{Q \in \mathcal{F}} \rho(\ell(Q)) m_{3 Q}(g f) \chi_{Q}(x) \\
& \quad \leq C \sum_{Q \in \mathcal{F}_{1}}\left(\varepsilon \tilde{\rho}(\ell(Q)) \chi_{Q}(x)+\tilde{\rho}\left(\ell\left(Q_{1}\right)\right) m_{3 Q_{1}}(g f) \chi_{Q_{1}}(x)\right) .
\end{aligned}
$$

Since $\tilde{\rho}(t)^{a-1} t^{-n}$ is nonincreasing,

$$
\varepsilon \tilde{\rho}(\ell(Q))|Q|^{1-1 / p}=\tilde{\rho}(\ell(Q))|Q|^{1-1 / p} \frac{\tilde{\rho}\left(\ell\left(Q_{0}\right)\right)^{a-1}}{\left|Q_{0}\right|^{n}} \leq \frac{\tilde{\rho}(\ell(Q))^{a}}{|Q|^{1 / p}} .
$$

This means that $\varepsilon \tilde{\rho}(\ell(Q)) \chi_{Q}(x)$ becomes a $\left(p^{\prime}, \tilde{\rho}^{a}\right)$-block. Thus, we know that $F_{1}$ can be majorized by the finite sum of the $\left(p^{\prime}, \tilde{\rho}^{a}\right)$-blocks and conclude that $F_{1} \in \mathcal{B}^{p^{\prime}, \tilde{\rho}^{a}}$. 
The case $i=2$. For every cube $Q \in \mathcal{D}_{2}\left(Q_{0}\right)$ we have

$$
\rho(\ell(Q)) m_{3 Q}(g f) \chi_{Q}(x)=\frac{\rho(\ell(Q))}{\tilde{\rho}(\ell(Q))^{a}} \int_{Q_{0}} g(y) f(y) d y \cdot \tilde{\rho}(\ell(Q))^{a} \frac{\chi_{Q}(x)}{|Q|} .
$$

Because $\tilde{\rho}(\ell(Q))^{a} \frac{\chi_{Q}(x)}{|Q|}$ becomes a $\left(p^{\prime}, \tilde{\rho}^{a}\right)$-block, we shall compute

$$
\int_{Q_{0}} g(y) f(y) d y \sum_{\nu=1+\log _{2} \ell\left(Q_{0}\right)}^{\infty} \frac{\rho\left(2^{\nu}\right)}{\tilde{\rho}\left(2^{\nu}\right)^{a}} .
$$

It follows from $q>r$ and (3.2) that

$$
\begin{aligned}
\int_{Q_{0}} g(y) f(y) d y & =\left|Q_{0}\right| m_{Q_{0}}(g f) \leq\left|Q_{0}\right| m_{Q_{0}}^{(r)}(g) m_{Q_{0}}^{\left(r^{\prime}\right)}(f) \\
& \leq\left|Q_{0}\right| m_{Q_{0}}^{(q)}(g) m_{Q_{0}}^{\left(r^{\prime}\right)}(f) \leq \tilde{\rho}\left(\ell\left(Q_{0}\right)\right)^{a-1} .
\end{aligned}
$$

The fact that $\tilde{\rho}$ is nondecreasing and (1.7) yield that

$$
\begin{aligned}
\sum_{\nu=1+\log _{2} \ell\left(Q_{0}\right)}^{\infty} \frac{\rho\left(2^{\nu}\right)}{\tilde{\rho}\left(2^{\nu}\right)^{a}} & \leq \int_{\ell\left(Q_{0}\right)}^{\infty} \tilde{\rho}(s)^{-a} \frac{\rho(s)}{s} d s \\
& =\int_{\ell\left(Q_{0}\right)}^{\infty} \frac{d}{d s}\left(\frac{1}{1-a} \tilde{\rho}(s)^{1-a}\right) d s \\
& \leq \frac{1}{a-1} \tilde{\rho}\left(\ell\left(Q_{0}\right)\right)^{1-a} .
\end{aligned}
$$

Multiplying both sides, we obtain (3.4) $\leq C$, and hence $F_{2} \in \mathcal{B}^{p^{\prime}, \tilde{\rho}^{a}}$. The proof of the theorem is now complete.

Remark 3.6. We do not know that if a function $f$ satisfies

$$
\sup _{w}\left|\int_{\mathbb{R}^{n}} f(x) w(x) d x\right|<\infty
$$

where the supremum is taken over all the normalized $w \in \mathcal{M}^{p, \phi}$, then $f$ belongs to $\mathcal{B}^{p^{\prime}, \phi}$.

We finish the section by restating Theorem 3.1 and Corollary 3.2 in terms of the fractional integral operator $I_{\alpha}$. The results hold by letting $\rho(t) \equiv t^{n \alpha}, a \alpha=1 / p_{0}$ and $b \alpha=1 / q_{0}$.

Proposition 3.7. Let $0<\alpha<1,1<p \leq p_{0}<\infty, 1<q \leq q_{0} \leq \infty$ and $1<r \leq r_{0}<\infty$. Suppose that $q>r, 1 / p_{0}>\alpha, 1 / q_{0} \leq \alpha, 1 / r_{0}=1 / q_{0}+1 / p_{0}-\alpha$ and $r / r_{0}=p / p_{0}$. Then

$$
\left\|I_{\alpha}(g f)\right\|_{\mathcal{B}^{p^{\prime}, p_{0}^{\prime}}} \leq C\|g\|_{\mathcal{M}^{q, q_{0}}}\|f\|_{\mathcal{B}^{r^{\prime}, r_{0}^{\prime}}}
$$

if $g$ is a continuous function.

Proposition 3.8. Let $0<\alpha<1,1<p \leq p_{0}<\infty$ and $1<r \leq r_{0}<\infty$. Suppose that $1 / p_{0}>\alpha, 1 / r_{0}=1 / p_{0}-\alpha$ and $r / r_{0}=p / p_{0}$. Then

$$
\left\|I_{\alpha} f\right\|_{\mathcal{B}^{p^{\prime}, p_{0}^{\prime}}} \leq C\|f\|_{\mathcal{B}^{r^{\prime}, r_{0}^{\prime}}} .
$$


Remark 3.9. In Proposition 3.8, if $r / r_{0}=p / p_{0}$ is replaced by $1 / r=1 / p-\alpha$, then, using the Hardy-Littlewood-Sobolev inequality locally and taking care of the larger scales in the same manner as in the proof of Theorem 3.1, one has a naive bound for $I_{\alpha}$.

\section{Sharpness of the Results}

Finally in this paper, from two points of view, we shall discuss the sharpness of the results for the fractional integral operator (the Riesz potential) $I_{\alpha}$. First, we examine the optimality of the Fefferman-Phong inequality.

$$
\begin{gathered}
\text { Let } 0<\alpha<1 \text {. If } 1<p \leq p_{0}<\infty \text { and } p<q \leq 1 / \alpha \text {, then } \\
\qquad g \cdot I_{\alpha} f\left\|_{\mathcal{M}^{p, p_{0}}} \leq C\right\| g\left\|_{\mathcal{M}^{q, 1 / \alpha}}\right\| f \|_{\mathcal{M}^{p, p_{0}}} .
\end{gathered}
$$

For the fractional integral operator $I_{\alpha}$ inequality (4.1) is crucial in the following sense: Since one has (cf. [19, p. 118])

$$
I_{\alpha}\left(I_{\beta} f\right)=I_{\alpha+\beta} f, \quad \alpha, \beta>0, \alpha+\beta<1,
$$

we obtain, when $1<q \leq q_{0}, 1<r \leq r_{0}, q>r$ and $\alpha-1 / q_{0}>0$,

$$
\left\|g \cdot I_{\alpha} f\right\|_{\mathcal{M}^{r, r_{0}}} \leq C\|g\|_{\mathcal{M}^{q, q_{0}}}\left\|I_{\alpha-1 / q_{0}} f\right\|_{\mathcal{M}^{r, r_{0}}} .
$$

This and the Adams theorem (the boundedness of the Riesz potential in the classical Morrey spaces) yield Proposition 1.8. We remark that, instead of (4.2), we have verified Theorem 1.3 for the generalized fractional integral operator $T_{\rho}$. We shall prove the following.

Proposition 4.1. Let $1<r \leq r_{0}<\infty$ and $r<1 / \alpha$. Then, for any $c>0$ we can find positive measurable functions $f$ and $g$ such that

$$
\left\|g \cdot I_{\alpha} f\right\|_{\mathcal{M}^{r, r_{0}}}>c\|g\|_{\mathcal{M}^{r, 1 / \alpha}}\|f\|_{\mathcal{M}^{r, r_{0}}} .
$$

This proposition can be proved by using the method developed in [4]. Although the Fefferman-Phong inequality is elementary and has important applications, the results corresponding to the Lebesgue spaces cannot be found in the literature as far as we know. In (4.1), if one replaces the fractional integral operator $I_{\alpha}$ by the fractional maximal operator $M_{\alpha}$, then it holds when $q=p$ (see Theorem 1.7 and Lemma 2.4).

Proof. If the claim is not true, then, by noticing that the block space $\mathcal{B}^{r^{\prime}, r_{0}^{\prime}}$ is the predual space of the Morrey space $\mathcal{M}^{r, r_{0}}$ (see the previous section), there exists a constant $c_{0}>0$ such that

$$
\left|\int_{\mathbb{R}^{n}} h(x) \cdot g(x) \cdot I_{\alpha} f(x) d x\right| \leq c_{0}\|h\|_{\mathcal{B}^{r^{\prime}, r_{0}^{\prime}}}\|g\|_{\mathcal{M}^{r, 1 / \alpha}}\|f\|_{\mathcal{M}^{r, r_{0}}} .
$$

Let $0<\delta<1$ be the solution to the equation

$$
\left(\frac{2}{1-\delta}\right)^{\alpha}(1-\delta)^{1 / r}=1
$$

and let $N$ be a positive large integer.

$$
\begin{gathered}
\text { Let } E_{0}=Q_{0,1}=\left[0,\left(\frac{2}{1-\delta}\right)^{N}\right]^{n} \text {. Delete from } Q_{0,1} \text { all but an open middle cube } \\
P_{0,1} \text {, of side } \delta\left(\frac{2}{1-\delta}\right)^{N} \text {, and the } 2^{n} \text { closed corner cubes } Q_{1, j} \text {, of side }\left(\frac{2}{1-\delta}\right)^{N-1},
\end{gathered}
$$


to obtain $E_{1}=\bigcup_{j=1}^{2^{n}} Q_{1, j}$ and $F_{0}=P_{0,1}$. Continue in this way $N$ steps: At the $k<N$ stage replace each cube of $E_{k-1}$ by an open middle cube $P_{k-1, j}$, of side $\delta\left(\frac{2}{1-\delta}\right)^{N-k+1}$, and the $2^{n}$ closed corner cubes $Q_{k, j}$, of side $\left(\frac{2}{1-\delta}\right)^{N-k}$, to obtain $E_{k}=\bigcup_{j=1}^{2^{n k}} Q_{k, j}$ and $F_{k-1}=\bigcup_{j=1}^{2^{n(k-1)}} P_{k-1, j}$. Thus $E_{N}$ contains $2^{n N}$ closed unit cubes and $F_{k}, k=0,1, \cdots, N-1$, contains $2^{n k}$ open cubes of side $\delta\left(\frac{2}{1-\delta}\right)^{N-k}$. Then, we have the following:

$$
\frac{\left|E_{N} \cap Q_{k, j}\right|}{\left|Q_{k, j}\right|}=(1-\delta)^{n(N-k)}, \quad k=0,1, \cdots, N
$$

and

$$
\left|Q_{k, j}\right|^{\alpha}\left(\frac{\left|E_{N} \cap Q_{k, j}\right|}{\left|Q_{k, j}\right|}\right)^{1 / r}=1, \quad k=0,1, \cdots, N,
$$

where we have used (4.4).

We first let $g(x)=\chi_{E_{N}}(x)$. Then it follows from (4.6) and simple geometric observation that

$$
\|g\|_{\mathcal{M}^{r, 1 / \alpha}} \leq C .
$$

It also follows from (4.4) and (4.5) that

$$
\begin{aligned}
& \left|Q_{0,1}\right|^{1 / r_{0}^{\prime}}\left(\frac{\left|E_{N}\right|}{\left|Q_{0,1}\right|}\right)^{1 / r^{\prime}} \\
& \quad=\left|E_{N}\right|\left|Q_{0,1}\right|^{-1 / r_{0}}\left(\frac{\left|E_{N}\right|}{\left|Q_{0,1}\right|}\right)^{-1 / r} \\
& =2^{n N}\left|Q_{0,1}\right|^{\alpha-1 / r_{0}}
\end{aligned}
$$

This yields that $\left(2^{n N}\left|Q_{0,1}\right|^{\alpha-1 / r_{0}}\right)^{-1} g$ is an $\left(r^{\prime}, r_{0}^{\prime}\right)$-block. Since the characteristic function of a unit cube is also an $\left(r^{\prime}, r_{0}^{\prime}\right)$-block, we obtain

$$
\|g\|_{\mathcal{B}^{r^{\prime}, r_{0}^{\prime}}} \leq \begin{cases}2^{n N}\left|Q_{0,1}\right|^{\alpha-1 / r_{0}}, & r_{0} \alpha<1 \\ 2^{n N}, & r_{0} \alpha \geq 1\end{cases}
$$

For $x \in E_{N}$ we clearly have $I_{\alpha} g(x) \geq C$. For $x \in F_{k}, k=0,1, \cdots, N-1$, noting that there exists a unique cube $Q_{k, j}$ containing $x$, we have

$$
I_{\alpha} g(x) \geq C\left|Q_{k, j}\right|^{\alpha} \frac{\left|E_{N} \cap Q_{k, j}\right|}{\left|Q_{k, j}\right|}=C\left(\left(\frac{2}{1-\delta}\right)^{\alpha}(1-\delta)\right)^{n(N-k)} .
$$

These yield $I_{\alpha} g(x) \geq C u(x)$, provided that

$$
u(x)=g(x)+\sum_{k=0}^{N-1}\left(\left(\frac{2}{1-\delta}\right)^{\alpha}(1-\delta)\right)^{n(N-k)} \chi_{F_{k}}(x) .
$$


It follows by using (4.4) again that

$$
\left(\left(\frac{2}{1-\delta}\right)^{\alpha}(1-\delta)\right)^{r^{\prime}}=\left(\left(\frac{2}{1-\delta}\right)^{r \alpha}(1-\delta)^{r}\right)^{1 /(r-1)}=1-\delta
$$

and by using

$$
\left|F_{k}\right|=2^{n k} \cdot \delta^{n}\left(\frac{2}{1-\delta}\right)^{n(N-k)}
$$

that

$$
\int_{\mathbb{R}^{n}} u(x)^{r^{\prime}} d x=2^{n N}\left(1+N \delta^{n}\right) .
$$

Similarly, we see that

$$
\int_{Q_{k, j}} u(x)^{r^{\prime}} d x=2^{n(N-k)}\left(1+(N-k) \delta^{n}\right), \quad k=0,1, \cdots, N .
$$

Finally, we wish to prove

$$
\left\|u^{1 /(r-1)}\right\|_{\mathcal{M}^{r, r_{0}}} \leq \begin{cases}\left(1+N \delta^{n}\right)^{1 / r}\left|Q_{0,1}\right|^{1 / r_{0}-\alpha}, & r_{0} \alpha<1 \\ \left(1+N \delta^{n}\right)^{1 / r}, & r_{0} \alpha \geq 1\end{cases}
$$

To this end, it suffices to check only for the cubes $Q_{k, j}$ and $P_{k, j}$. Since, by simple geometric observation, the average of $u^{1 /(r-1)}$ over all cubes $Q \in \mathcal{Q}, Q \subset Q_{0,1}$ and $\ell(Q) \geq 1$ can be controlled by the averages of that over cubes $Q_{k, j}$ and $P_{k, j}$. Hence,

$$
\begin{aligned}
\left|Q_{k, j}\right|^{\frac{1}{r_{0}}-\frac{1}{r}}\left(\int_{Q_{k, j}} u(x)^{r^{\prime}} d x\right)^{1 / r} \\
=\left|Q_{k, j}\right|^{1 / r_{0}-\alpha}\left|Q_{k, j}\right|^{\alpha-1 / r}\left(2^{n(N-k)}\left(1+(N-k) \delta^{n}\right)\right)^{1 / r} \\
=\left(2^{n(N-k)}\right)^{-1 / r}\left|Q_{k, j}\right|^{1 / r_{0}-\alpha}\left|Q_{k, j}\right|^{\alpha}(1-\delta)^{n(N-k) / r} \\
\quad \times\left(2^{n(N-k)}\left(1+(N-k) \delta^{n}\right)\right)^{1 / r} \\
=\left|Q_{k, j}\right|^{1 / r_{0}-\alpha}\left(1+(N-k) \delta^{n}\right)^{1 / r}
\end{aligned}
$$

where we have used (4.9) to obtain

$$
\begin{aligned}
& \left|P_{k, j}\right|^{\frac{1}{r_{0}}-\frac{1}{r}}\left(\int_{P_{k, j}} u(x)^{r^{\prime}} d x\right)^{1 / r} \\
& =\left(\delta^{n}\right)^{\frac{1}{r_{0}}-\frac{1}{r}}\left|Q_{k, j}\right|^{\frac{1}{r_{0}}-\frac{1}{r}}\left(2^{n(N-k)} \delta^{n}\right)^{1 / r} \\
& =\left(\delta^{n}\right)^{\frac{1}{r_{0}}-\frac{1}{r}}\left(2^{n(N-k)}\right)^{-1 / r}\left|Q_{k, j}\right|^{1 / r_{0}-\alpha}\left(2^{n(N-k)} \delta^{n}\right)^{1 / r} \\
& =\delta^{n / r_{0}}\left|Q_{k, j}\right|^{1 / r_{0}-\alpha} .
\end{aligned}
$$

These imply (4.10).

Letting $f(x)=u(x)^{1 /(r-1)}$ and $h(x)=g(x)$ in (4.3), using

$$
\int_{\mathbb{R}^{n}} h(x) g(x) I_{\alpha} f(x) d x \geq C \int_{\mathbb{R}^{n}} u(x)^{r^{\prime}} d x=C 2^{n N}\left(1+N \delta^{n}\right),
$$

we obtain $\left(1+N \delta^{n}\right)^{1 / r^{\prime}} \leq C \cdot c_{0}$ by (4.7), (4.8) and (4.10). This is impossible for large $N$.

Second, we shall prove that Proposition 1.8 is sharp in the following sense. 
Proposition 4.2. Let $0<\alpha<1,1<p<p_{0}<\infty, 1<q_{0}<\infty$ and $1<r<$ $r_{0}<\infty$. Suppose that $1 / r_{0}=1 / q_{0}+1 / p_{0}-\alpha$ and $r / r_{0}=p / p_{0}$. Then for every $0<\varepsilon \leq r_{0}-r$, there exist $f \in \mathcal{M}^{p, p_{0}}$ and $g \in \mathcal{M}^{q_{0} p / p_{0}, q_{0}}$ such that

$$
\left\|g \cdot I_{\alpha} f\right\|_{\mathcal{M}^{r+\varepsilon, r_{0}}}=\infty .
$$

Proof. Let $1<s<s_{0}, 1 / s_{0}=1 / p_{0}-\alpha$ and $s / s_{0}=p / p_{0}$. Then we can select an $f \in \mathcal{M}^{p, p_{0}}$ that satisfies

$$
\left\|I_{\alpha} f\right\|_{\mathcal{M}^{s, s_{0}}}=1 \text { but }\left\|I_{\alpha} f\right\|_{\mathcal{M}^{s+\varepsilon s_{0} / r_{0}, s_{0}}}=\infty .
$$

This is justified by Theorem 10 in 15 .

First, a simple arithmetic shows that

$$
\|g\|_{\mathcal{M}^{q_{0} p / p_{0}, q_{0}}}=1, \quad g \equiv\left(I_{\alpha} f\right)^{s_{0} / q_{0}}
$$

and

It then follows that

$$
r\left(1+\frac{s_{0}}{q_{0}}\right)=r s_{0}\left(\frac{1}{s_{0}}+\frac{1}{q_{0}}\right)=s_{0} \frac{r}{r_{0}}=s .
$$

$$
\begin{aligned}
\| g & \cdot I_{\alpha} f \|_{\mathcal{M}^{r+\varepsilon, r_{0}}} \\
= & \sup _{Q \in \mathcal{Q}}\left(\frac{1}{|Q|^{1-(r+\varepsilon) / r_{0}}} \int_{Q} I_{\alpha} f(x)^{(r+\varepsilon)\left(1+s_{0} / q_{0}\right)} d x\right)^{1 /(r+\varepsilon)} \\
= & \sup _{Q \in \mathcal{Q}}\left(\frac{1}{|Q|^{1-s / s_{0}-\varepsilon / r_{0}}} \int_{Q} I_{\alpha} f(x)^{s+\varepsilon s_{0} / r_{0}} d x\right)^{1 /(r+\varepsilon)} \\
= & \left(\left\|I_{\alpha} f\right\|_{\mathcal{M}^{s+\varepsilon s_{0} / r_{0}, s_{0}}}\right)^{r /(r+\varepsilon)}=\infty .
\end{aligned}
$$

Therefore, the proof is now complete.

\section{REFERENCES}

[1] David R. Adams, A note on Riesz potentials, Duke Math. J., 42 (1975), 765-778. MR0458158 $(56: 16361)$

[2] David R. Adams and J. Xiao, Nonlinear potential analysis on Morrey spaces and their capacities, Indiana Univ. Math. J., 53 (2004), 1629-1663. MR2106339 (2005h:31015)

[3] F. Chiarenza and M. Frasca, Morrey spaces and Hardy-Littlewood maximal function, Rend. Mat., 7 (1987), 273-279. MR.985999 (90f:42017)

[4] Maja J. Carro, C. Pf́rez, F. Soria and J. Soria, Maximal functions and the control of weighted inequalities for the fractional integral operator, Indiana Univ. Math. J., 54 (2005), no. 3, $627-$ 644. MR.2151228 (2006f:42016)

[5] D. Cruz-Uribe, SFO and C. Pérez, Two-weight, weak-type norm inequalities for fractional integrals, Calderón-Zygmund operators and commutators, Indiana Univ. Math. J., 49 (2000), 697-721. MR1793688 (2001i:42021)

[6] Eridani, On the boundedness of a generalized fractional integral on generalized Morrey spaces, Tamkang J. Math., 33 (2002), 335-340. MR1936313 (2003j:42011)

[7] Eridani and H. Gunawan, Stummel class and Morrey spaces, Southeast Asian Bull. Math., 29 (2005), 1053-1056. MR2195380 (2006i:46054)

[8] Eridani, H. Gunawan and E. Nakai, On generalized fractional integral operators, Sci. Math. Jpn., 60 (2004), 539-550. MR2099586 (2006a:47044)

[9] C. Fefferman, The uncertainty principle, Bull. Amer. Math. Soc., 9 (1983), 129-206. MR707957 (85f:35001)

[10] D. Gilbarg and N. S. Trudinger, Elliptic Partial Differential Equations of Second Order, 2nd ed., Springer-Verlag, Berlin, 1983. MR737190 (86c:35035)

[11] E. Nakai, The Hardy-Littlewood maximal operator, singular integral operators and the Riesz potentials on generalized Morrey spaces, Math. Nachr., 166 (1994), 95-103. MR1273325 (95k:42030) 
[12] _ Generalized fractional integrals on Orlicz-Morrey spaces, Banach and function spaces, 323-333, Yokohama Publ., Yokohama, 2004. MR2146936 (2006c:42016)

[13] _ On generalized fractional integrals on the weak Orlicz spaces, $\mathrm{BMO}_{\phi}$, the Morrey spaces and the Campanato spaces, Function spaces, interpolation theory and related topics (Lund, 2000), 389-401, de Gruyter, Berlin, 2002. MR1943296 (2003k:26014)

[14] , On generalized fractional integrals, Taiwanese J. Math., 5 (2001), 587-602. MR 1849780 (2002f:47071)

[15] P. Olsen, Fractional integration, Morrey spaces and Schrödinger equation, Comm. Partial Differential Equations, 20 (1995), 2005-2055. MR1361729 (97a:35042)

[16] O. Blasco, A. Ruiz and L. Vega, Non-interpolation in Morrey-Campanato and block spaces, Ann. Scuola Norm. Sup. Pisa Cl. Sci. 28 (1999), 31-40. MR.1679077 (2000c:46048)

[17] C. Pérez, Sharp L L ${ }^{p}$-weighted Sobolev inequalities, Ann. Inst. Fourier (Grenoble), 45 (1995), 809-824. MR1340954 (96m:42032)

[18] Y. Sawano, Generalized Morrey spaces for non-doubling measures, Non-linear Differential Equations and Applications, 15 (2008), 413-425. MR2465971(2010d:42035)

[19] E. M. Stein, Singular Integrals and Differentiability Properties of Functions, Princeton University Press, Princeton, 1970. MR0290095 (44:7280)

[20] S. Sugano and H. Tanaka, Boundedness of fractional integral operators on generalized Morrey spaces, Sci. Math. Jpn., 58 (2003), 531-540. MR2017532 (2004k:42026)

[21] H. Tanaka, Morrey spaces and fractional operators, J. Aust. Math. Soc., 88 (2010), no. 2, 247-259. MR2629934 (2011d:42061)

[22] C. T. Zorko, Morrey space, Proc. Amer. Math. Soc., 98 (1986), 586-592. MR861756 (88b:46062)

Department of Mathematics, Kyoto University, Kitasirakawa, Sakyoku, Kyoto, 6068502, JAPAN

E-mail address: yosihiro@math.kyoto-u.ac.jp

Department of Mathematics, Kobe City College of Technology, 8-3 Gakuen-higashiMACHI, Nishi-KU, KoBe 651-2194, JAPAN

E-mail address: sugano@kobe-kosen.ac.jp

Graduate School of Mathematical Sciences, The University of Tokyo, 3-8-1 Komaba, Meguro-ku, TOKYO 153-8914, JAPAN

E-mail address: htanaka@ms.u-tokyo.ac.jp 